Article

\title{
Evaluation of Systemic Renin and Angiotensin II Levels in Normal Tension Glaucoma
}

\author{
Soo Ji Jeon ${ }^{1}$, Hyung Bin Hwang ${ }^{2}$ and Na Young Lee ${ }^{1, *}$ \\ 1 Department of Ophthalmology, Eunpyeong St. Mary's Hospital, College of Medicine, The Catholic \\ University of Korea, Seoul 03312, Korea; sj8801@gmail.com \\ 2 Department of Ophthalmology, Incheon St. Mary's Hospital, College of Medicine, The Catholic University \\ of Korea, Incheon 21431, Korea; leoanzel@catholic.ac.kr \\ * Correspondence: nyny5555@naver.com; Tel.: +82-2-2030-2795; Fax: +82-2-599-7405
}

Received: 27 October 2020; Accepted: 24 November 2020; Published: 26 November 2020

check for updates

\begin{abstract}
The purpose of this study was to investigate the function of the renin-angiotensinaldosterone system (RAAS) in normal tension glaucoma (NTG) patients by measuring the level of renin and angiotensin II (AngII) in the plasma. Twenty-four patients with NTG and 38 control subjects were included in this study. Renin and AngII were measured in the blood samples of all subjects by enzyme-linked immunosorbent assay (ELISA). No significant differences were found in the complete blood count, fasting glucose, low-density lipoprotein (LDL), and high-sensitivity C-reactive protein (hs-CRP) levels between the control and NTG groups. The systemic concentration and variability of the renin concentration in the blood was significantly higher in the NTG group ( $p=0.005$ and 0.005 , respectively). According to multivariate logistic regression analysis, the variability of the renin concentration was associated with NTG $(p=0.006)$. In conclusion, the systemic concentration and variability of renin levels were elevated in NTG patients. An altered renin concentration could represent a difference in RAAS function in NTG patients.
\end{abstract}

Keywords: renin; angiotensin II; normal tension glaucoma; renin-angiotensin-aldosterone system

\section{Introduction}

Glaucoma is characterized by the progressive death of retinal ganglion cells (RGCs) and the associated visual field (VF) defect. Elevated intraocular pressure (IOP) is traditionally known to be the main cause of glaucoma [1]. However, some glaucoma patients with normal IOP have shown similar clinical features to patients with elevated IOP. In Asia, glaucoma patients with normal IOP are the predominant type [2,3].

Glaucoma patients with normal IOP, known as normal tension glaucoma (NTG), have distinct clinical features associated with vascular dysregulation. According to several studies, orthostatic hypotension, systemic arterial hypotension, Raynaud's phenomenon, and migraines are common in NTG patients [4-6]. According to a population-based study, fluctuation of systolic blood pressure (BP) is a contributing factor to the development of open angle glaucoma (OAG) [7]. Considering that this study was conducted in Korea, where most OAG patients have normal IOP, this result also supports the association of systemic hemodynamic instability with NTG.

The renin-angiotensin-aldosterone system (RAAS) is important for regulating hemodynamic stability and fluid volume in the body [8]. When hypotension occurs with dysregulated vascular status, the RAAS is activated and renin is released from the kidneys. The released renin acts on angiotensinogen to generate angiotensin II (AngII), which is the physiologically active form [9]. AngII, which is mediated via AngII receptors, has multiple effects, including helping to maintain a constant 
fluid volume in the body $[10,11]$. Therefore, we hypothesized that NTG patients with a high probability of hemodynamic instability could have an altered RAAS function compared to normal controls.

The purpose of this study was to investigate the systemic hemodynamic features associated with RAAS function in patients with NTG. To the best of our knowledge, no study has been conducted measuring renin and AngII levels in the blood of NTG patients. We measured the concentration of renin and AngII, as well as the complete blood count, fasting glucose, total cholesterol, high-density lipoprotein (HDL), and low-density lipoprotein (LDL) levels in the blood of the study subjects. The results of the plasma concentrations of renin and AngII, including the variability, were compared to those of normal controls.

\section{Materials and Methods}

\subsection{Study Design and Population}

This study was performed according to the tenets of the Declaration of Helsinki and was approved by the Institutional Review and Ethics Board of Incheon St. Mary's Hospital, South Korea. All subjects of this single-center, case-control study provided written, informed consent. Normal controls were recruited from a routine ocular examination group, and patients with NTG were recruited from the glaucoma clinic of Incheon St. Mary's Hospital. Patients were excluded if they met any of the following criteria: (1) a history of non-glaucomatous optic neuropathy; (2) a history of eye trauma or surgery, except for uncomplicated cataract extraction; (3) pathologic myopia (i.e., chorioretinal atrophy, intrachoroidal cavitation, choroidal neovascularization, and lacquer crack); (4) other retinal diseases, including diabetic retinopathy and retinal vascular diseases such as vascular occlusion or uveitis.

All subjects underwent comprehensive ophthalmic examinations, including best-corrected visual acuity (BCVA), refraction, Goldmann applanation tonometry, slit-lamp examination, gonioscopy, and dilated fundus bimicroscopy. Fundus photography, stereoscopic photography of the optic disc (Kowa, VK-2, Torrance, CA, USA), and perimetry with the Swedish Interactive Threshold Algorithm (SITA) 24-2 test on a Humphrey Field Analyzer were performed to diagnose NTG. The diagnostic criteria for NTG were as follows: (1) open angle by gonioscopy; (2) IOP <22 mmHg; (3) glaucomatous-appearing optic disc morphology corresponding to glaucomatous VF defects on SITA 24-2 results, as follows: a glaucomatous-appearing optic disc accompanied by increased cup-disc ratio (CDR) of more than 0.7 , a difference in vertical CDR of more than 0.2 between both eyes, retinal nerve fiber layer (RNFL) defects, or neural rim thinning. We defined glaucomatous VF defects as meeting two of the following three criteria: (1) a cluster of 3 points with a probability lower than $5 \%$ on the pattern deviation map in at least one hemifield and including at least 1 point with a probability lower than $1 \%$ or a cluster of 2 points with a probability lower than 1\%; (2) glaucoma hemifield test results outside normal limits; (3) a pattern standard deviation outside $95 \%$ of the normal limits. VF defects were confirmed by at least two consecutive and reliable tests (defined as a false-negative rate of $<15 \%$, a false-positive rate of $<15 \%$, and fixation losses of $<20 \%$ ). All NTG patients had anti-glaucomatic eyedrops and showed stable disease status without VF progression for three years.

$\mathrm{BP}$ was measured after the study subjects had rested in a sitting position for $5 \mathrm{~min}$. Trained clinicians took measurements of two consecutive systolic and diastolic BPs, and the average value was used. For hypertension patients, the type of hypertensive medication was identified, and subjects with angiotensin-converting enzyme (ACE) inhibitors or angiotensin II receptor blockers (ARBs) were excluded, as these medications could interfere with systemic renin and angiotensin II levels.

\subsection{Measurement of Renin and Angiotensin II with Enzyme-Linked Immunosorbent Assay (ELISA)}

After fasting for $12 \mathrm{~h}$ and resting for $30 \mathrm{~min}$, blood samples for the biochemical analysis were obtained by standard venipuncture. Blood was collected into tubes containing EDTA as an anticoagulant for plasma preparation. As soon as a blood sample was collected, the tube was frozen at $-20^{\circ} \mathrm{C}$, and repeated freeze-thaw cycles were avoided. Frozen samples were thawed before conducting ELISA 
of renin (R\&D Systems, Minneapolis, MN, USA) and EIA of AngII (Phoenix Pharmaceuticals, Inc., Burlingame, CA, USA) using commercially available kits and according to the manufacturer's instructions. In brief, standards, samples, and controls were added to each well of a microplate pre-coated with a secondary antibody specific for renin and AngII. After incubation and washing the plate, a primary antibody was added, and the plate was again incubated and washed. Subsequently, a chromogenic substrate was added to each well for the development of color, and the intensity was measured by a microplate reader. All measurements were assayed in duplicate and the mean values were calculated. In addition, the standard deviations (SDs) of the measurements were calculated to assess the variability of renin and AngII.

\subsection{Statistical Analysis}

All data are expressed as mean \pm standard deviation. Independent $t$-tests were used to compare the continuous variables between the control and NTG groups. Chi-square tests were used to compare the categorical variables between the two groups. To identify the factors associated with NTG, logistic regression analyses were performed. Variables with $p$-values $<0.1$ in the univariate analyses were included in the multivariate analysis. The odds ratio (OR) and $95 \%$ confidence interval (CI) were calculated for each variable. All statistical analyses were performed with SPSS version 24.0 (IBM Corp., Armonk, NY, USA). $p<0.05$ was considered statistically significant.

\section{Results}

This study included 24 patients with NTG and 38 controls as study subjects. Table 1 shows the clinical characteristics of the subjects. There were no significant differences in age, gender, the ratio of hypertension and diabetes, or blood pressure between the two groups. Ocular characteristics such as BCVA, spherical equivalent, and IOP did not differ significantly between the two groups.

Table 1. Comparison of the clinical characteristics of the control group and the normal tension glaucoma (NTG) group $(n=62)$.

\begin{tabular}{cccc}
\hline Clinical Characteristics & Control $(n=38)$ & NTG $(n=24)$ & $p$-Value \\
\hline Age (years) & $67.97( \pm 10.85)$ & $67.61( \pm 15.58)$ & 0.920 \\
Sex (male/female) & $15 / 23$ & $14 / 10$ & 0.147 \\
Hypertension (\%) & $12(31.6)$ & $11(45.8)$ & 0.258 \\
Diabetes (\%) & $8(21.1)$ & $2(8.3)$ & 0.185 \\
BCVA (decimal) & $0.77( \pm 0.25)$ & $0.71( \pm 0.27)$ & 0.297 \\
Spherical equivalent (diopter) & $-1.05( \pm 1.56)$ & $-1.95( \pm 2.64)$ & 0.162 \\
Intraocular pressure (mmHg) & $12.94( \pm 2.89)$ & $12.41( \pm 3.61)$ & 0.526 \\
Systolic blood pressure (mmHg) & $131.28( \pm 18.72)$ & $128.17( \pm 9.17)$ & 0.425 \\
Diastolic blood pressure (mmHg) & $75.17( \pm 9.39)$ & $76.58( \pm 7.93)$ & 0.595 \\
\hline
\end{tabular}

BCVA: best corrected visual acuity.

The laboratory findings were comparable between the two groups, as shown in Table 2. White blood cell (WBC) count, red blood cell (RBC) count, hemoglobin, hematocrit, platelet count, fasting glucose, LDL, and hs-CRP were not significantly different between the control and NTG groups. With respect to the lipid profile, total cholesterol was higher $(p=0.027)$ and HDL was lower $(p=0.036)$ in the NTG patients.

The mean concentration and SD of renin and AngII from each group are reported in Table 3 and Figure 1. The mean concentration and SD of renin were higher in the NTG group (mean: $528.06 \pm 341.54$ vs. $1355.64 \pm 1279.52, p=0.005$; SD: $17.79 \pm 14.29$ vs. $49.45 \pm 49.41, p=0.005)$. The mean concentration and SD of AngII were higher in the NTG group but not statistically significant $(p=0.121$ and 0.089 , respectively). According to Figure 1, the range of distribution of renin was wider in the NTG group. 
Table 2. Comparison of the blood examination of the control group and the normal tension glaucoma (NTG) group.

\begin{tabular}{cccc}
\hline Examination Results & Control $(\boldsymbol{n}=\mathbf{3 8})$ & NTG $(\boldsymbol{n}=\mathbf{2 4})$ & $\boldsymbol{p}$-Value \\
\hline WBC $\left(10^{9} / \mathrm{L}\right)$ & $6.37( \pm 2.54)$ & $7.33( \pm 3.01)$ & 0.184 \\
RBC $\left(10^{12} / \mathrm{L}\right)$ & $4.36( \pm 0.47)$ & $4.26( \pm 0.60)$ & 0.474 \\
Hemoglobin $(\mathrm{g} / \mathrm{dL})$ & $13.44( \pm 1.28)$ & $13.13( \pm 1.71)$ & 0.429 \\
Hematocrit $(\%)$ & $40.41( \pm 3.45)$ & $39.71( \pm 5.07)$ & 0.557 \\
Platelet $\left(10^{9} / \mathrm{L}\right)$ & $256.28( \pm 83.46)$ & $257.62( \pm 69.89)$ & 0.948 \\
Fasting glucose $(\mathrm{mg} / \mathrm{dL})$ & $116.25( \pm 28.66)$ & $104.39( \pm 16.20)$ & 0.070 \\
Total cholesterol $(\mathrm{mg} / \mathrm{dL})$ & $170.78( \pm 38.69)$ & $199.41( \pm 49.81)$ & $\mathbf{0 . 0 2 7}$ \\
HDL $(\mathrm{mg} / \mathrm{dL})$ & $52.14( \pm 7.53)$ & $42.50( \pm 1.73)$ & $\mathbf{0 . 0 3 6}$ \\
LDL $(\mathrm{mg} / \mathrm{dL})$ & $108.53( \pm 34.21)$ & $112.00( \pm 34.49)$ & 0.729 \\
Hs-CRP $(\mathrm{mg} / \mathrm{dL})$ & $1.62( \pm 2.83)$ & $3.58( \pm 5.52)$ & 0.092 \\
\hline
\end{tabular}

WBC: white blood cell; RBC: red blood cell; HDL: high-density lipoprotein; LDL: low-density lipoprotein; hs-CRP: high-sensitivity C-reactive protein. Significant values are shown in bold.

Table 3. Results of the enzyme-linked immunosorbent assay (ELISA) of renin and angiotensin II in the control group and the normal tension glaucoma (NTG) group.

\begin{tabular}{cccc}
\hline ELISA results & Control $(\boldsymbol{n}=\mathbf{3 8})$ & NTG $(\boldsymbol{n}=\mathbf{2 4})$ & $\boldsymbol{p}$-Value \\
\hline Renin_mean $(\mathrm{pg} / \mathrm{mL})$ & $528.06( \pm 341.54)$ & $1355.64( \pm 1279.52)$ & $\mathbf{0 . 0 0 5}$ \\
Renin_SD & $17.79( \pm 14.29)$ & $49.45( \pm 49.41)$ & $\mathbf{0 . 0 0 5}$ \\
Angiotensin II_mean (ng/mL) & $0.14( \pm 0.38)$ & $0.91( \pm 2.31)$ & 0.121 \\
Angiotensin II_SD & $0.01( \pm 0.01)$ & $0.05( \pm 0.13)$ & 0.089
\end{tabular}

SD: standard deviation. Significant values are shown in bold.
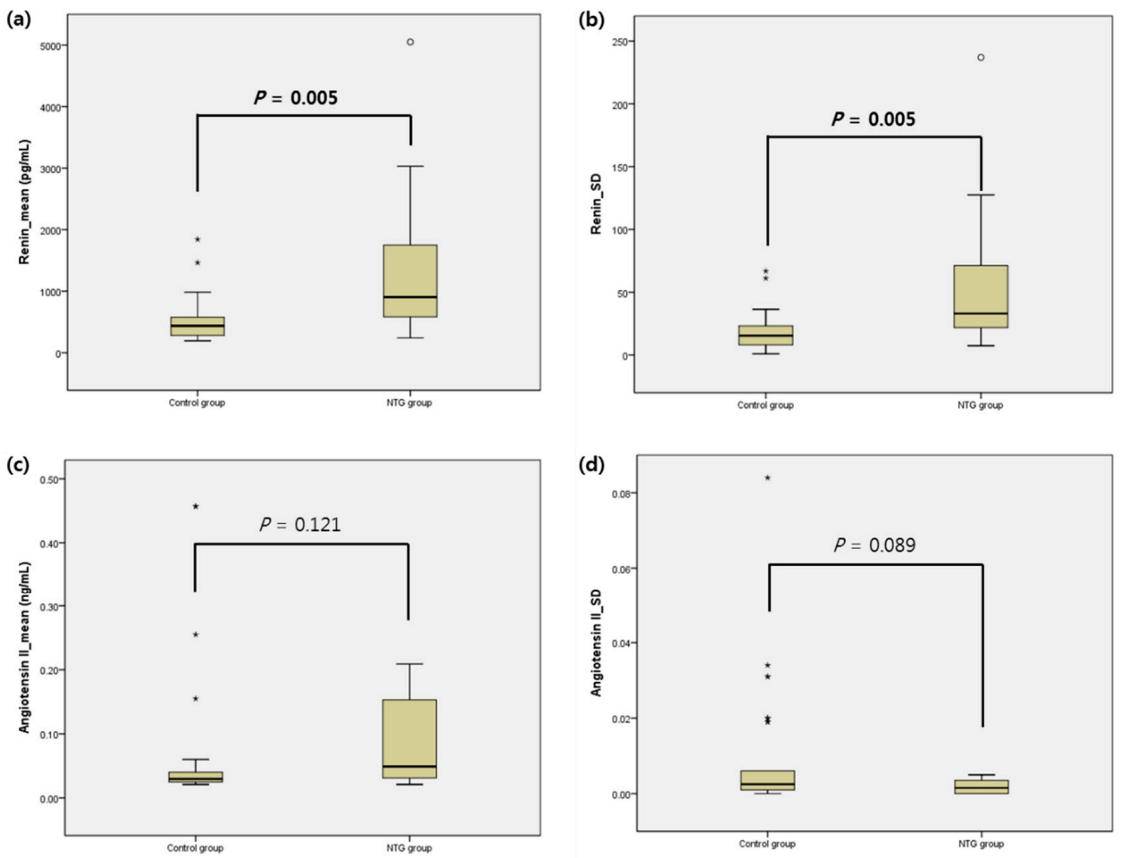

Figure 1. Graphs representing the mean value and standard deviation (SD) of renin and angiotensin II from the plasma of the control and NTG groups. The midline of the box plot represents the mean, while the bar of each box shows the $95 \%$ confidential interval. Dots and stars identify the outliers. Outliers of the mean and SD of angiotensin II in the NTG group could not be marked in the graph due to a limitation of scale. In angiotensin II, the mean values of the outliers were 1.84, 3.82, and 10.5. In angiotensin II, the SD values of outliers were $0.516,0.322,0.328$, and 0.225 . Statistical significance was compared to the control group and significant values are shown in bold. (a) Renin_mean (pg/mL), (b) Renin_SD, (c) Angiotensin II_mean (ng/mL), (d) Angiotensin II_SD. 
The factors related to NTG from the clinical and laboratory findings were evaluated using logistic regression analyses (Table 4 ). In the univariate analyses, the total cholesterol, mean value, and SD of renin were associated with NTG ( $p=0.033,0.003$, and 0.002 , respectively). In the multivariate analysis, including the variables with $p$-values $\leq 0.10$ from the univariate analyses, only the SD of renin was significantly associated with NTG $(p=0.006)$.

Table 4. Systemic factors associated with normal tension glaucoma (NTG).

\begin{tabular}{|c|c|c|c|c|c|c|}
\hline \multirow{2}{*}{ Variables } & \multicolumn{3}{|c|}{ Univariate } & \multicolumn{3}{|c|}{ Multivariate } \\
\hline & $\operatorname{Exp}(B)$ & $95 \%$ CI & $p$-Value & $\operatorname{Exp}(\beta)$ & $95 \%$ CI & $p$-Value \\
\hline Age (years) & 0.998 & 0.953 to 1.044 & 0.918 & & & \\
\hline Female gender (vs. male) & 0.466 & 0.165 to 1.318 & 0.466 & & & \\
\hline Hypertension & 1.833 & 0.638 to 5.264 & 0.260 & & & \\
\hline Diabetes & 0.341 & 0.066 to 1.765 & 0.200 & & & \\
\hline Systolic BP & 0.987 & 0.950 to 1.026 & 0.515 & & & \\
\hline Diastolic BP & 1.019 & 0.953 to 1.089 & 0.587 & & & \\
\hline WBC & 1.138 & 0.935 to 1.383 & 0.197 & & & \\
\hline $\mathrm{RBC}$ & 0.693 & 0.258 to 1.864 & 0.468 & & & \\
\hline Platelet & 1.000 & 0.994 to 1.007 & 0.947 & & & \\
\hline Total cholesterol & 1.015 & 1.001 to 1.029 & 0.033 & 1.017 & 1.000 to 1.034 & 0.052 \\
\hline Fasting glucose & 0.977 & 0.951 to 1.004 & 0.101 & & & \\
\hline HDL & 0.634 & 0.311 to 1.295 & 0.211 & & & \\
\hline LDL & 1.003 & 0.986 to 1.020 & 0.723 & & & \\
\hline Hs-CRP & 1.126 & 0.971 to 1.305 & 0.117 & & & \\
\hline Renin_mean & 1.002 & 1.001 to 1.003 & 0.003 & & & \\
\hline Renin_SD & 1.058 & 1.021 to 1.096 & 0.002 & 1.051 & 1.015 to 1.088 & 0.006 \\
\hline Angiotensin II_mean & 1.951 & 0.838 to 4.544 & 0.121 & & & \\
\hline Angiotensin II_SD & 1.093 & 0.980 to 1.219 & 0.110 & & & \\
\hline
\end{tabular}

BP: blood pressure; WBC: white blood cell; RBC: red blood cell; HDL: high-density lipoprotein; LDL: low-density lipoprotein; hs-CRP: high-sensitivity C-reactive protein; SD: standard deviation. Significant values are shown in bold.

\section{Discussion}

The systemic features representing hemodynamic instability include systemic arterial hypotension, orthostatic hypotension, and a nocturnal BP dip. These features have been reported as commonly found characteristics in NTG patients [12-14]. A population-based study reported that lower systolic $\mathrm{BP}$ doubles the risk of glaucoma development, independent of the effect of IOP [15]. According to the study of Choi et al., a nocturnal BP dip is associated with circadian ocular perfusion pressure disturbance, and disturbed ocular perfusion could affect the development of NTG [13]. Park et al. suggested that orthostatic hypotension is a significant factor associated with NTG progression [16].

When systemic hypotension occurs in the body, the RAAS starts to regulate the blood volume and systemic vascular resistance [17]. The RAAS functions to maintain the blood pressure through elevating the arterial tone and blood volume. In response to lowered BP, activated juxtaglomerular cells of the kidney cleave prorenin to renin. Increased renin causes the sequential cleavage of angiotensinogen to angiotensin I and II [18].

In the present study, we found that the systemic mean concentration and variability of renin was higher in the NTG patients. Additionally, the SD of the renin concentration was a meaningful factor associated with NTG. AngII was thought to be elevated in response to an increase in renin; however, there was no statistically significant difference between the NTG and control groups. In short, this study identified a link between systemic RAAS functioning and NTG through the measurement of the systemic concentration of renin and AngII. RAAS function showed a different pattern in the NTG patients when compared to normal controls.

According to our previous study, the systemic concentration of endothelin-1 (ET-1) and macrophage chemoattractant protein-1 (MCP-1) are elevated in NTG patients [19]. ET-1 is widely known as a mediator of inflammation, contributing to vascular dysfunction [20]. The chemotactic activity of MCP-1 can also cause diverse vasculopathy by diapedesis of monocytes [21]. These factors associated with 
vascular dysfunction might explain the disturbed fluid volume and RAAS function, leading to the rise of renin and angiotensin II levels, to some extent.

In healthy eyes, retinal blood flow is mediated by endothelial cells because there is no autonomic nervous system innervation [22]. AngII, affected by the release of renin as a constituent of the RAAS, is one of the mediators controlling the contraction and relaxation of vascular endothelium [23]. A previous study using animal models reported that angiotensin plays a role in the regulation of ophthalmic microcirculation [24]. A weakened blood-brain barrier of the optic nerve head in glaucoma [25] could pass systemic RAAS components and may have an influence on the retinal microcirculation.

In addition, most RAAS components and receptors were found in the retinal tissue. The local RAAS from tissue, including the retina, has been reported as a system that maintains the homeostasis of tissue [26]. Prorenin receptors that could result in renin having enzymatic activity were reported as important molecules in the RAAS of tissue. Activated renin and the resultant angiotensin elevation could function as a stimulator of inflammatory cascades in the neural tissue of the retina [27]. There have been several studies reporting the role of neuroinflammation in glaucoma [28-30], and the RAAS could be a link between vascular dysregulation and neuroinflammation-associated RGC death in glaucoma.

In this study, total cholesterol and HDL were different between the two groups. The NTG group had worse lipid profiles in terms of dyslipidemia. However, lipid profiles were not independently associated with NTG in the regression analyses. Indeed, there is controversy regarding the relationship between dyslipidemia and glaucoma. Wang et al. suggested that hyperlipidemia is related to an increased risk of glaucoma and IOP elevation [31]. It has also been reported that higher serum cholesterol is related to a higher risk of glaucoma [32]. However, according to a population-based study in Korea, there is no significant association of total cholesterol and HDL with glaucoma [33]. Our study is limited due to the small number of subjects; thus, future studies should be conducted with larger numbers of subjects and various populations.

The other limitations of this study should be mentioned. First, clinical features associated with vascular dysregulation, such as optic disc hemorrhage, migraine, and Raynaud's phenomenon, were not included in our analysis. Since normal controls were recruited from routine medical examinations, information on their hemodynamic features was not available. Several studies have already reported the correlation of vascular dysregulation with optic disc hemorrhage [34], migraines [35], and Raynaud's phenomenon [36]. For further understanding, it is necessary to study these comprising factors and to investigate their effect on RAAS function in NTG patients. Second, since we included only subjects with NTG, it is hard to generalize these results to all types of glaucoma. High-pressure glaucoma has different hemodynamic systemic characteristics to NTG [37]; therefore, it is unreasonable to extend the distinct RAAS status to patients with other types of glaucoma. Furthermore, because all subjects had normal IOP, it was hard to determine the effect of IOP on RAAS function. For further understanding, studies comprising glaucoma patients with elevated IOP should be performed with a larger number of subjects. Finally, the short half-life degradation time of AngII could have prevented the accurate measurement of the plasma. Though freezing was performed immediately after the blood sample was taken to minimize measurement errors, the sample could still have been affected by the half-life time. This could be a compromising factor that resulted in the nonsignificant results for AngII in contrast to renin.

In conclusion, NTG patients showed elevation and fluctuation of systemic renin concentration. The difference in RAAS function in NTG patients may be associated with diverse features of vascular dysregulation. Further studies investigating the relationship between RAAS and NTG could suggest a novel approach for disease modulation.

Author Contributions: S.J.J. wrote the main manuscript text. H.B.H. analyzed the data and prepared the tables. N.Y.L. provided the main concept of the study and reviewed the manuscripts. All authors have read and agreed to the published version of the manuscript. 
Funding: This research was supported by a Grant of Translational R\&D Project through the Institute for Bio-Medical Convergence, Incheon St. Mary's Hospital, The Catholic University of Korea. This research received no external funding.

Conflicts of Interest: The authors report no conflicts of interest. The authors alone are responsible for the content and writing of the paper.

\section{References}

1. Weinreb, R.N.; Khaw, P.T. Primary open-angle glaucoma. Lancet 2004, 363, 1711-1720. [CrossRef]

2. Iwase, A.; Suzuki, Y.; Araie, M.; Yamamoto, T.; Abe, H.; Shirato, S.; Kuwayama, Y.; Mishima, H.K.; Shimizu, H.; Tomita, G.; et al. The prevalence of primary open-angle glaucoma in Japanese: The Tajimi Study. Ophthalmology 2004, 111, 1641-1648. [CrossRef]

3. Kim, C.S.; Seong, G.J.; Lee, N.H.; Song, K.C.; Namil Study Group, Korean Glaucoma Society. Prevalence of primary open-angle glaucoma in central South Korea the Namil study. Ophthalmology 2011, 118, 1024-1030. [CrossRef] [PubMed]

4. Mudumbai, R.C. Clinical update on normal tension glaucoma. Semin. Ophthalmol. 2013, 28, 173-179. [CrossRef]

5. Kosior-Jarecka, E.; Wrobel-Dudzinska, D.; Lukasik, U.; Zarnowski, T. Ocular and Systemic Risk Factors of Different Morphologies of Scotoma in Patients with Normal-Tension Glaucoma. J. Ophthalmol. 2017, 2017, 1480746. [CrossRef]

6. Galassi, F.; Giambene, B.; Varriale, R. Systemic vascular dysregulation and retrobulbar hemodynamics in normal-tension glaucoma. Investig. Ophthalmol. Vis. Sci. 2011, 52, 4467-4471. [CrossRef]

7. Lee, N.Y.; Jung, Y.; Han, K.; Park, C.K. Fluctuation in systolic blood pressure is a major systemic risk factor for development of primary open-angle glaucoma. Sci. Rep. 2017, 7, 43734. [CrossRef]

8. Jia, G.; Aroor, A.R.; Hill, M.A.; Sowers, J.R. Role of Renin-Angiotensin-Aldosterone System Activation in Promoting Cardiovascular Fibrosis and Stiffness. Hypertension 2018, 72, 537-548. [CrossRef]

9. Sequeira Lopez, M.L.; Pentz, E.S.; Nomasa, T.; Smithies, O.; Gomez, R.A. Renin cells are precursors for multiple cell types that switch to the renin phenotype when homeostasis is threatened. Dev. Cell 2004, 6, 719-728. [CrossRef]

10. Szczepanska-Sadowska, E.; Czarzasta, K.; Cudnoch-Jedrzejewska, A. Dysregulation of the Renin-Angiotensin System and the Vasopressinergic System Interactions in Cardiovascular Disorders. Curr. Hypertens. Rep. 2018, 20, 19. [CrossRef]

11. Pacurari, M.; Kafoury, R.; Tchounwou, P.B.; Ndebele, K. The Renin-Angiotensin-Aldosterone System in Vascular Inflammation and Remodeling. Int. J. Inflamm. 2014, 2014, 689360. [CrossRef] [PubMed]

12. Sung, K.R.; Cho, J.W.; Lee, S.; Yun, S.C.; Choi, J.; Na, J.H.; Lee, Y.; Kook, M.S. Characteristics of visual field progression in medically treated normal-tension glaucoma patients with unstable ocular perfusion pressure. Investig. Ophthalmol. Vis. Sci. 2011, 52, 737-743. [CrossRef] [PubMed]

13. Choi, J.; Jeong, J.; Cho, H.S.; Kook, M.S. Effect of nocturnal blood pressure reduction on circadian fluctuation of mean ocular perfusion pressure: A risk factor for normal tension glaucoma. Investig. Ophthalmol. Vis. Sci. 2006, 47, 831-836. [CrossRef] [PubMed]

14. Choi, J.; Kim, K.H.; Jeong, J.; Cho, H.S.; Lee, C.H.; Kook, M.S. Circadian fluctuation of mean ocular perfusion pressure is a consistent risk factor for normal-tension glaucoma. Investig. Ophthalmol. Vis. Sci. 2007, 48, 104-111. [CrossRef]

15. Leske, M.C.; Wu, S.Y.; Hennis, A.; Honkanen, R.; Nemesure, B. Risk factors for incident open-angle glaucoma: The Barbados Eye Studies. Ophthalmology 2008, 115, 85-93. [CrossRef]

16. Park, H.Y.; Park, S.H.; Park, C.K. Central visual field progression in normal-tension glaucoma patients with autonomic dysfunction. Investig. Ophthalmol. Vis. Sci. 2014, 55, 2557-2563. [CrossRef]

17. Nehme, A.; Zouein, F.A.; Zayeri, Z.D.; Zibara, K. An Update on the Tissue Renin Angiotensin System and Its Role in Physiology and Pathology. J. Cardiovasc. Dev. Dis. 2019, 6, 14. [CrossRef]

18. Ren, L.; Lu, X.; Danser, A.H.J. Revisiting the Brain Renin-Angiotensin System-Focus on Novel Therapies. Curr. Hypertens. Rep. 2019, 21, 28. [CrossRef]

19. Lee, N.Y.; Park, H.Y.; Park, C.K.; Ahn, M.D. Analysis of systemic endothelin-1, matrix metalloproteinase-9, macrophage chemoattractant protein-1, and high-sensitivity C-reactive protein in normal-tension glaucoma. Curr. Eye Res. 2012, 37, 1121-1126. [CrossRef] 
20. Freeman, B.D.; Machado, F.S.; Tanowitz, H.B.; Desruisseaux, M.S. Endothelin-1 and its role in the pathogenesis of infectious diseases. Life Sci. 2014, 118, 110-119. [CrossRef]

21. Niu, J.; Kolattukudy, P.E. Role of MCP-1 in cardiovascular disease: Molecular mechanisms and clinical implications. Clin. Sci. 2009, 117, 95-109. [CrossRef] [PubMed]

22. Luo, X.; Shen, Y.M.; Jiang, M.N.; Lou, X.F.; Shen, Y. Ocular Blood Flow Autoregulation Mechanisms and Methods. J. Ophthalmol. 2015, 2015, 864871. [CrossRef] [PubMed]

23. Lesk, M.R.; Wajszilber, M.; Deschenes, M.C. The effects of systemic medications on ocular blood flow. Can. J. Ophthalmol. 2008, 43, 351-355. [CrossRef] [PubMed]

24. Meyer, P.; Flammer, J.; Luscher, T.F. Local action of the renin angiotensin system in the porcine ophthalmic circulation: Effects of ACE-inhibitors and angiotensin receptor antagonists. Investig. Ophthalmol. Vis. Sci. 1995, 36, 555-562.

25. Grieshaber, M.C.; Flammer, J. Does the blood-brain barrier play a role in Glaucoma? Surv. Ophthalmol. 2007, 52 (Suppl. 2), S115-S121. [CrossRef]

26. Soubrier, F.; Alhenc-Gelas, F.; Hubert, C.; Allegrini, J.; John, M.; Tregear, G.; Corvol, P. Two putative active centers in human angiotensin I-converting enzyme revealed by molecular cloning. Proc. Natl. Acad. Sci. USA 1988, 85, 9386-9390. [CrossRef]

27. Kurihara, T.; Ozawa, Y.; Ishida, S.; Okano, H.; Tsubota, K. Renin-Angiotensin system hyperactivation can induce inflammation and retinal neural dysfunction. Int. J. Inflam. 2012, 2012, 581695. [CrossRef]

28. Soto, I.; Howell, G.R. The complex role of neuroinflammation in glaucoma. Cold Spring Harb. Perspect. Med. 2014, 4, a017269. [CrossRef]

29. Williams, P.A.; Marsh-Armstrong, N.; Howell, G.R. Neuroinflammation in glaucoma: A new opportunity. Exp. Eye Res. 2017, 157, 20-27. [CrossRef]

30. Mac Nair, C.E.; Nickells, R.W. Neuroinflammation in Glaucoma and Optic Nerve Damage. Prog. Mol. Biol. Transl. Sci. 2015, 134, 343-363.

31. Wang, S.; Bao, X. Hyperlipidemia, Blood Lipid Level, and the Risk of Glaucoma: A Meta-Analysis. Investig. Ophthalmol. Vis. Sci. 2019, 60, 1028-1043. [CrossRef] [PubMed]

32. Kang, J.H.; Boumenna, T.; Stein, J.D.; Khawaja, A.; Rosner, B.A.; Wiggs, J.L.; Pasquale, L.R. Association of Statin Use and High Serum Cholesterol Levels With Risk of Primary Open-Angle Glaucoma. JAMA Ophthalmol. 2019, 137, 756-765. [CrossRef] [PubMed]

33. Shon, K.; Sung, K.R. Dyslipidemia, Dyslipidemia Treatment, and Open-angle Glaucoma in the Korean National Health and Nutrition Examination Survey. J. Glaucoma 2019, 28, 550-556. [CrossRef] [PubMed]

34. Grieshaber, M.C.; Terhorst, T.; Flammer, J. The pathogenesis of optic disc splinter haemorrhages: A new hypothesis. Acta Ophthalmol. Scand. 2006, 84, 62-68. [CrossRef] [PubMed]

35. Panconesi, A.; Bartolozzi, M.L.; Guidi, L. Migraine pain: Reflections against vasodilatation. J. Headache Pain 2009, 10, 317-325. [CrossRef]

36. Herrick, A.L. Pathogenesis of Raynaud's phenomenon. Rheumatology 2005, 44, 587-596. [CrossRef]

37. Song, B.J.; Caprioli, J. New directions in the treatment of normal tension glaucoma. Indian J. Ophthalmol. 2014, 62, 529-537.

Publisher's Note: MDPI stays neutral with regard to jurisdictional claims in published maps and institutional affiliations.

(C) 2020 by the authors. Licensee MDPI, Basel, Switzerland. This article is an open access article distributed under the terms and conditions of the Creative Commons Attribution (CC BY) license (http://creativecommons.org/licenses/by/4.0/). 